\begin{tabular}{|c|l|}
\hline Title & Gel biomachine based on muscle proteins \\
\hline Author(s) & Kwon, Hyuck Joon; Shikinaka, Kazuhiro; Kakugo, A kira; Gong, Jian Ping; O sada, Y oshihito \\
\hline Citation & $\begin{array}{l}\text { Polymer Bulletin, 58(1), 43-52 } \\
\text { https://doi.org/10.1007/300289-006-0613-4 }\end{array}$ \\
\hline Issue Date & 2007-01 \\
\hline Doc URL & http://hdl.handle.net/2115/26274 \\
\hline Rights & The original publication is available at www.springerlink.com \\
\hline Type & article (author version) \\
\hline File Information & PB58-1.pdf \\
\hline
\end{tabular}

Instructions for use 


\title{
Gel biomachine based on muscle proteins
}

\author{
Hyuck Joon Kwon ${ }^{1}$, Kazuhiro Shikinaka ${ }^{1}$, Akira Kakugo ${ }^{1}$, \\ Jian Ping Gong ${ }^{1,2}\left(\right.$ (D), Yoshihito Osada ${ }^{1}$
}

${ }^{1}$ Biological Sciences, Graduate School of Science, Hokkaido University, Sapporo 060-0810, Japan, ${ }^{2}$ SORST, JST,

E-mail: gong@sci.hokudai.ac.jp Fax:+81-11-706-2774

\section{Summary}

We have created an ATP fueled soft gel machine reconstructed from muscle proteins. Chemically cross-linked gels of polymer-actin complex, several decade times the length of native actin filament (Factin) move on myosin coated surface with a velocity as high as that of native F-actin, by coupling to ATP hydrolysis. The motility observed in muscle protein-gels suggests that one might reconstruct a soft machine fueled by chemical energy by using actin and myosin molecules as elementary elements. We have investigated the growth process of polymer-actin complexes and the correlation between the polarity and the motility of polymer-actin complex gels.

\section{Introduction}

Biological motors have two basic differences from man-made machines. One is in their principles. The motion of a man-made machine, which is constructed from hard and dry materials such as metals, ceramics or plastics, is realized by the relative displacement of the macroscopic constituent parts of the machine. In contrast, the motion of biogical motors, which consists of soft and wet protein and tissues, is caused by a molecular deformation that is integrated to a macroscopic level through its hierarchical structure ${ }^{[1-3]}$. The other is in their energy sources. The man-made machine is fueled by electrical or thermal energy with an efficiency of around $30 \%$, but a biological motor is driven by direct conversion from chemical energy with an efficiency as high as $80-90 \%{ }^{[4]}$.

In order to create biomimetic systems, polymer gels have been employed due to their reversible size and shape change, thereby realizing the motion by integrating the deformation on a molecular level. Over the past number of years, using these idea, several kinds of 
artificial soft machines have been constructed using synthetic polymer gels $^{[5-11]}$. However, the lack of hierarchical structures and energy sources inside the gel lead to a decreased response and restricts the further application of such actuators for practical use in the human body. Recent advance in the field of molecular biology have enabled the discovery of a family of motor proteins and their assemblies. These proteins based on supramolecular assemblies would be biocompatible and practical for use in body. Actins and myosins are major component of muscle proteins and play an important role in dynamic motion of creatures that is caused by the molecular deformation using the chemical energy released by hydrolysis of ATP. Actins can form large complex in the presence of polycations due to the polyelectrolyte nature of F-actin.

We have found that chemically cross-linked gels of polymer-actin complexes can move on myosin coated surface with a velocity as high as that of native actins, by coupling to ATP hydrolysis. This result indicates that muscle proteins can be tailored into desired shape and size without sacrificing their bioactivities.

This article reviews recent work on the growth process of polymer-actin complexes in the presence of various polycations and the effect of the polarity of polymer-actin complexes on their velocities ${ }^{[12-15]}$. This study has illuminated the creation of ATP fueled biomachine with desired shape and polarity without loss of bioactivity.

\section{Results and discussion}

\section{Actin gel formed from polymer-actin complexes ${ }^{[13]}$}

Since the isoelectric point of actins is $\mathrm{pH} 4.7, \mathrm{~F}$-actins in neutral buffer are negatively charged. Therefore, they were assumed to form complexes with cationic polymers through electrostatic interaction. Figure 1a-d shows some examples of fluorescence microscope images of polymer-actin complexes obtained by mixing F-actins with p-Lys (Figure 1a) and x,y-ionene polymers (Figure 1b-1d) for $120 \mathrm{~min}$.

It has been shown that large filamentous, stranded and branched complexes of 20-30 $\mu \mathrm{m}$ in size are formed in the presence of p-Lys, 3,36,6- 6,12- ionene polymers and their morphological nature, both of size and shape, are strongly in contrast to that of native F-actin (Figure 1e). Figure $2 \mathrm{a}$ demonstrates time courses of the average length of the complexes in the presence of various kinds of polymers. Polymers have 


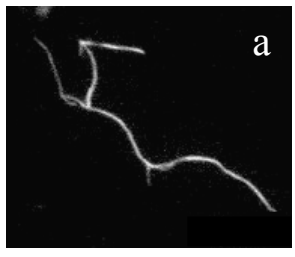

p-Lysine

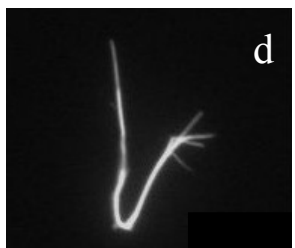

6,12-ionene

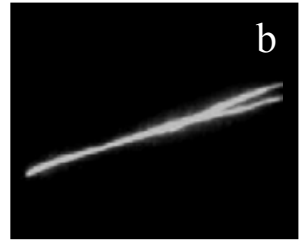

3,3-ionene

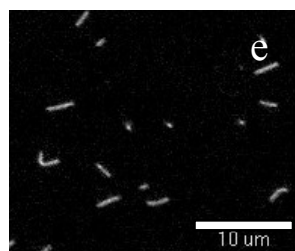

F-actin only

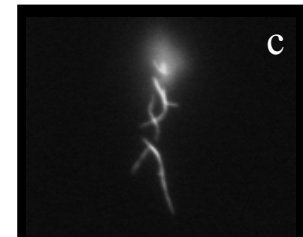

6,6-ionene

Figure 1 Fluorescence microscope images of polymer-actin complexes formed by mixing Factin and various cationic polymers at room temperature. (a) p-Lys, (b) 3,3-ionene, (c) 6,6ionene, (d) 6,12-ionene, (e) F-actin only. The molar ratio of ammonium cation of polymer to monomeric actin was kept constant at 30:1 for $\mathrm{x}$, y-ionene polymers and 100:1 for p-Lys. Actin concentration: $0.001 \mathrm{mg} / \mathrm{ml}$. Cited from ref. 13 and asking for permission.

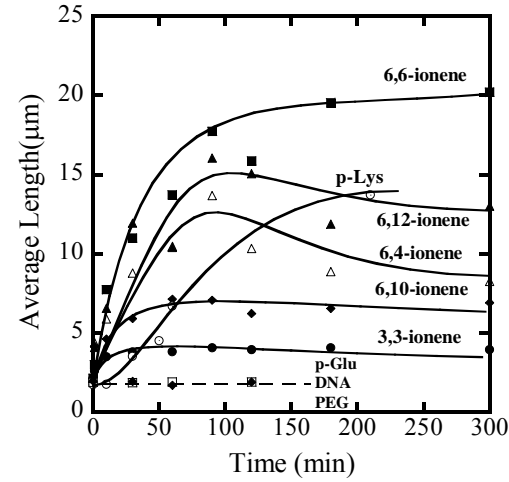

(a)

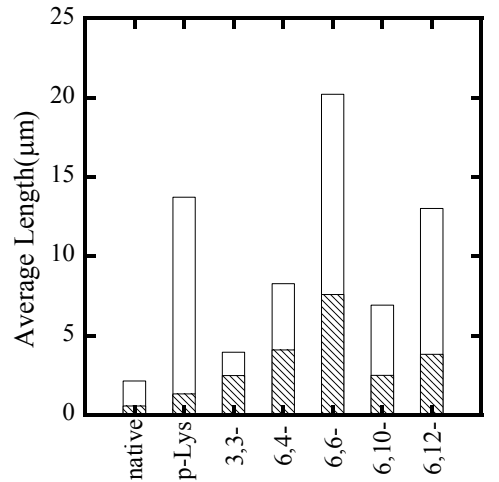

(b)

Figure 2 (a) Time courses of polymer-actin complexes growth. (b) Average length of polymeractin complexes observed from fluorescence microscope images (white columns) and from transmission electron microscopy(TEM) images(shade columns) at 210-300 min. The molar ratio of ammonium cation to monomeric actin was 30:1 for $\mathrm{x}, \mathrm{y}$-ionene polymers and 100:1 for p-Lys. Actin concentration: $0.001 \mathrm{mg} / \mathrm{ml}$. Cited from ref. 13 and asking for permission.

been mixed with following weight ratios keeping an actin concentration constant at $0.001 \mathrm{mg} / \mathrm{ml}$ as well as the molar ratio of ammonium cation of polymer to actin monomer of F-actin as 100:1 for p-Lys and 30:1 for 
ionene polymers. The number-average length of fluorescence image of F-actins is $2.14 \mu \mathrm{m}$ with a standard deviation of $0.11 \mu \mathrm{m}$ (average over 784 samples) in the F-buffer. However, polymer-actin complexes grow with time and reach as large as $5 \mu \mathrm{m}-20 \mu \mathrm{m}$ within one or two hours, which is about 2-10 times larger than that of native F-actin. The growth profiles depend on the chemical structure of the polycations. P-Lys shows a relatively slow growth profile but gives out a large complex. On the other hand, 3, 3-ionene polymer induces the formation of smallest complexes. These results indicate that hydrophobicity and charge density of the ionene polymers are important in complex formation. The average lengths of polymer-actin complexes are shown in Figure 2b. To confirm that this kind of actin growth is attributed to the complex formation by the electrostatic interaction between the negatively charged actins and cationic polymers, we further studied the actin growth in the mixture solution of actins and negatively charged polymers such as p-Glu, DNA, and neutral polymer, such as PEG at a molar ratio of monomeric units of polymer to F-actin of 100:1. As shown in Figure 2a, the F-actins do not grow into large filaments with time in the presence of these anionic or neutral polymers. Therefore, the electrostatic interaction between actins and cationic polymers should be essential for the formation of polymer-actin complexes. As shown in Figure 2a, although 3,3-ionene has a similar high charge density as that of p-Lys that has charged moiety on its side chain, it shows a much less ability of complex growth. This indicates that in spite of the initial formation of polymer-actin complex by the electrostatic interaction, the flexibility of the charged moiety would be an important factor. The complicated $\mathrm{x}, \mathrm{y}$ value dependence of the complex growth observed in x,y-ionene polymers might be associated with the complementary effect between the charge density and the flexibility of the charged moiety. Both 3,3-ionene and 6,10-ionene form short complexes because the former has a high charge density but with a less flexibility, while the latter has a high flexibility but with a low charge density. 6,6-ionene induces the formation of longest complex due to its proper charge density and flexibility. Here, we could not find a clear role related to the hydrophobicity of the $\mathrm{x}, \mathrm{y}$-ionene polymers. The decrease in the filament length after 100min of complex formation observed for 6,4- and 6,12-ionene complexes seems to be due to the aggregation of the complex.

Since the formation of polymer-actin complexes is an equilibrium reaction, he morphological features of the product should depend not 


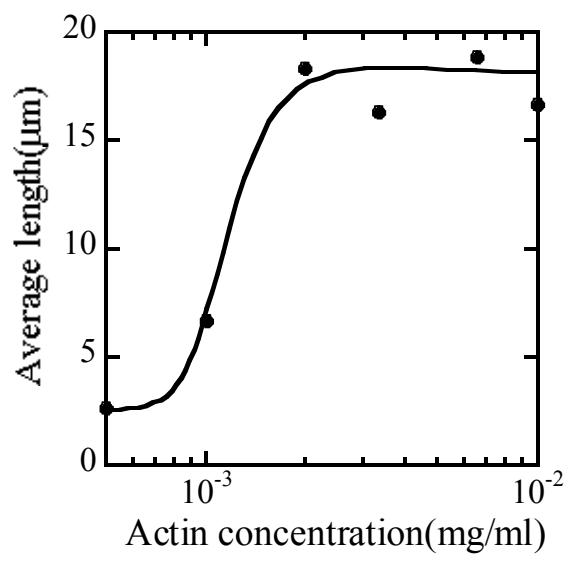

(a)

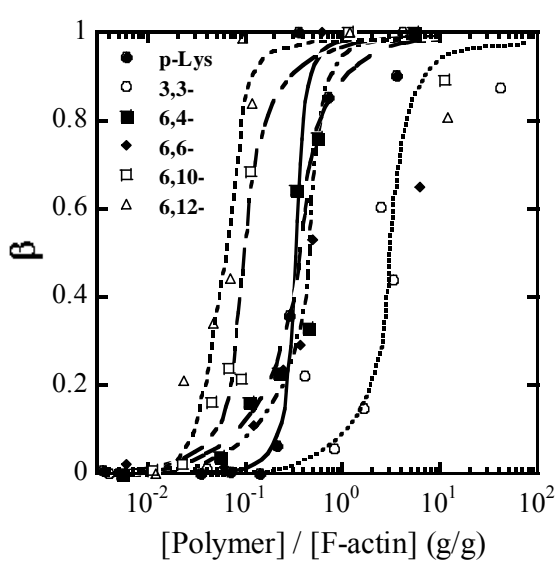

(b)

Figure 3 Average length of polymer-actin complexes as a function of F-actin concentration (a) and dependence of $\beta$ on the mixing ratio of polymer to actin (b). Here $\beta$ is defined as the ratio of average length to maximum length of polymer-actin complex. Data in (a) were obtained at $\left[\mathrm{p}\right.$-Lys] $=3.5 \times 10^{-4} \mathrm{mg} / \mathrm{ml}$ at $60 \mathrm{~min}$. and in (b) at an actin concentration of $0.001 \mathrm{mg} / \mathrm{ml}$ at 90 min. Cited from ref. 13 and asking for permission.

only on time and polymer structure, but also on concentrations of actins and polymers. Figure $3 \mathrm{a}$ shows the effect of actin concentration on the growth size of the polymer-actin complexes when mixed with p-Lys of a constant concentration. When the actin concentration exceeds $0.001 \mathrm{mg} / \mathrm{ml}$, the length of polymer-actin complexes increases steeply with the increase in the concentration and then saturate to a length around $15-20 \mu \mathrm{m}$. Figure $3 \mathrm{~b}$ shows the effect of $\mathrm{p}$-Lys concentration on the relative length $\beta$ of polymer-actin complexes in the equilibrium at a constant actin concentration $(0.001 \mathrm{mg} / \mathrm{ml})$. Here $\beta$ is defined as a ratio of average length to maximum length of polymer-actin complex. Only native F-actins are observed when the mixing ratio of p-Lys to actin is lower than $0.14 \mathrm{~g} / \mathrm{g}$, indicating that the polymer-actin complex does not form at such a low concentration. However, the length of polymer-actin complexes abruptly increases when the mixing ratio of p-Lys to F-actin exceeds $0.21 \mathrm{~g} / \mathrm{g}$. Thus, there exists a critical p-Lys concentration to form complex, indicating that the complex formation is cooperative. This cooperative behavior was also observed in the complex formation with ionene polymers, and the critical mixing ratios of 3,3-6,4- 6,66,10- and 6,12-ionene to F-actin were about 0.81, 0.054, 0.12, 0.024, and $0.022 \mathrm{~g} / \mathrm{g}$ at a constant F-actin concentration $(0.001 \mathrm{mg} / \mathrm{ml})$, 
respectively (Figure $3 b$ ). These results explain why we observed a shortest complex length of 3,3-ionene in Figure 2a performed at [3,3ionene $] /[$ actin $]=0.41 \mathrm{~g} / \mathrm{g}$, which was less than the critical value of $0.81 \mathrm{~g} / \mathrm{g}$. In conclusion, it is indicated that interaction between polymers and actins behave cooperatively, and polymer-actin complexes are formed only when both F-actin concentration and the mixing ratio exceed the critical values.

From Figure $3 \mathrm{~b}$ we can obtain the binding constant $(K)$ as well as the other thermodynamic parameters of the actin-polymer interaction by the following equation ${ }^{[16-18]} . K=K_{0} u=1 /(C s)_{0.5}$, where $K_{0}$ is the binding constant of the cationic polymer bound to an isolated binding site on the $\mathrm{F}$-actin (initiation process), $(\mathrm{Cs})_{0.5}$ is the cationic polymer concentration at $\beta=0.5$, here $\beta$ is defined as a ratio of average length to maximum length of polymer-actin complex, and $u$ is the cooperative parameter which tells the extra interaction energy between the binding sites (propagation process). The value of $u$ can be calculated from the slope of the growth profile at the half-length point.

$$
(d \beta / d \ln C s)_{0.5}=\sqrt{u} / 4 \quad(\text { Eq. } 2)
$$

$K_{0}$ and $u$ as well as the total binding energy $\left(\Delta F_{\text {total }}=-R T \ln K\right)$ and cooperative energy change $\left(\Delta F_{\text {coop }}=-R T \ln u\right)$ were calculated and the results are summarized in Table 1. One can see a large value of the cooperative parameter ( N ) and therefore a large cooperative energy $\left(\Delta F_{\text {coop }}\right)$ change for $\mathrm{p}$-Lys and 3,3-ionene, and the smallest value of cooperative parameter is observed for 6,12-ionene, which is one order of magnitude smaller than the is value of the other polymers. On the contrary, 6,12-ionene shows the highest binding constant of the initiation process $\left(K_{0}\right)$.

Table 1 Thermodynamic interaction parameters of complex formation between F-actin and various cationic polymers. Cited from ref. 13 and asking for permission.

\begin{tabular}{|c|c|c|c|c|c|}
\hline Polymer & $\begin{array}{c}K \\
{\left[10^{6}\right]}\end{array}$ & $u$ & $\begin{array}{c}K 0 \\
{\left[10^{5}\right]}\end{array}$ & $\begin{array}{c}\Delta \mathrm{F}_{\text {total }} \\
{\left[\mathrm{kJmol}^{-1}\right]}\end{array}$ & $\begin{array}{c}\Delta \mathrm{F}_{\text {coop }} \\
{\left[\mathbf{k J m o l}^{-1}\right]}\end{array}$ \\
\hline p-Lys & 3.9 & 31 & 1.2 & -37 & -8.4 \\
\hline 3,3 - ionene & 0.4 & 32 & 0.1 & -31 & -8.4 \\
\hline 6,4- & 2.9 & 4.4 & 6.6 & -36 & -3.5 \\
\hline $6,6-$ & 2.3 & 20 & 1.2 & -36 & -7.3 \\
\hline $6,10-$ & 10.6 & 7.0 & 1.5 & -39 & -4.7 \\
\hline $6,12-$ & 15.3 & 2.2 & 7.1 & -40 & -1.5 \\
\hline
\end{tabular}



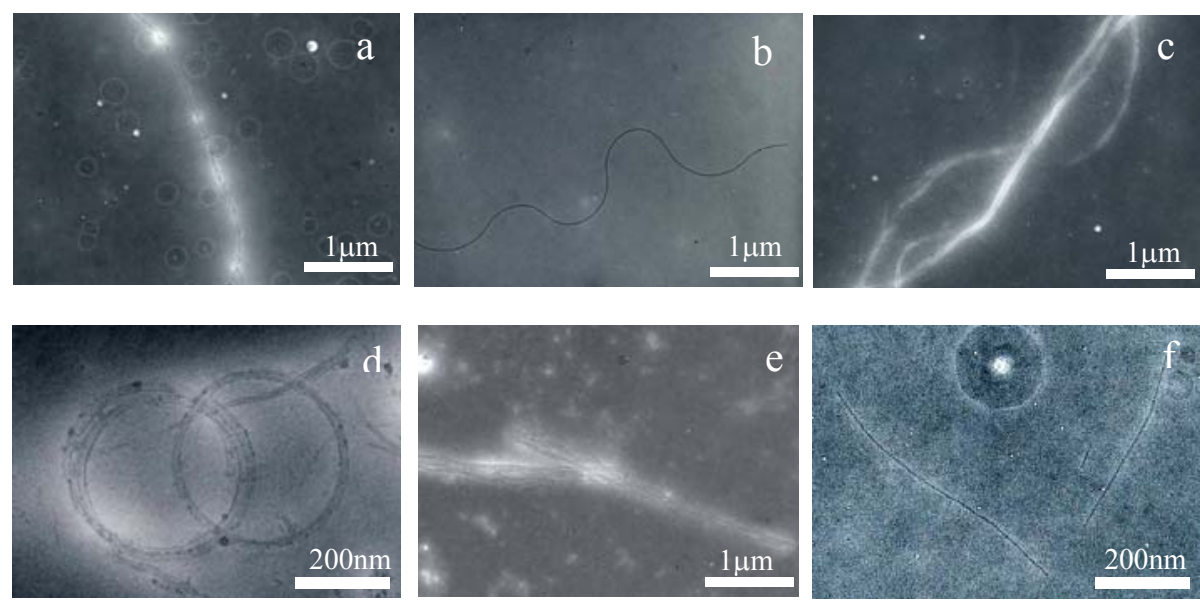

Figure 4 Transmission electron microscopy (TEM) images of polymer-actin complexes formed by mixing F-actin and various polymers at room temperature for 240 min. (a) p-Lys (b) 3,3ionene, (c) 6,6-ionene (d) 6,6-ionene (e) 3,3-ionene (f) F-actin only. Mixing molar ratios are 100:1, the same as fluorescence microscope observation (figure 1), except for (e) which is carried out at 300:1 ([3,3-ionene]/[Actin] $=4.1 \mathrm{~g} / \mathrm{g})$. Actin concentration: $0.001 \mathrm{mg} / \mathrm{ml}$. Cited from ref. 13 and asking for permission.

Since the lateral structures of the polymer-actin complexes are too small to be clearly observed by fluorescent microscope, we observed polymer-actin complexes by transmission electron microscopy (TEM), using the negative staining technique. Figure 4 shows the TEM images of the actin complexes prepared at a molar ratio of ammonium cation of polymer to monomeric actin as 100:1 for p-Lys and 30:1 for ionene polymers at a constant actin concentration $(0.001 \mathrm{mg} / \mathrm{ml})$. Figure $4 \mathrm{a}$ shows that F-actins form a relatively homogeneous and thin bundles in the presence of p-Lys. It is also found that an extremely homogeneous nano-scale wire (nano-wire) is formed with 3,3-ionene (Figure 4b). Filamentous complexes are observed in the presence of 6,6-ionene, (Figure 4c). Occasionally toroids like complex (nano-ring) was observed in the presence of 6,6-ionene (Figure 4d).

The average width of the p-Lys-actin complex is $21.0 \mathrm{~nm}$ with a standard deviation of $2.6 \mathrm{~nm}$. P-Lys-actin complexes are only slightly thicker than that of the native F-actin with almost the same width scattering. 3,3-ionene complexes also show a very thin and homogeneous wire-like morphology showing an average width of 16.1 $\mathrm{nm}$ with a standard deviation of $1.7 \mathrm{~nm}$. However, as shown in Figure $3 \mathrm{~b}$, the 3,3-ionene polymer concentration is still below the critical concentration at a molar ratio of ammonium cation to actin monomer as 
$30: 1$. Since the morphology of polymer-actin complexes is strongly dependent on the polymer concentration, we further investigated the 3,3-ionene-actin complex at a molar ratio of ammonium cation to actin monomer as 300:1 ([3,3-ionene $] /[$ Actin $]=4.1 \mathrm{~g} / \mathrm{g})$, which is above the critical concentration of complex formation. As shown in Figure 4e, thicker bundles are formed when the 3,3-ionene polymer exceeds the critical concentration. Similar morphology are observed for other $\mathrm{x}, \mathrm{y}-$ ionene polymers above their critical concentration. Actin-6,4-, 6,6-, 6,10-, and 6,12-ionene complexes have an average width of $79.0 \mathrm{~nm}$, $59.3 \mathrm{~nm}, 38.7 \mathrm{~nm}$ and $66.1 \mathrm{~nm}$ with a standard deviation of $60 \mathrm{~nm}, 29$ $\mathrm{nm}, 21 \mathrm{~nm}$, and $27 \mathrm{~nm}$ respectively. The polymer-actin complexes were chemically cross-linked with the cross-linking reagents to form stable gels (hereafter called, polymer-actin complex gel). The motility of these polymer-actin complex gels will be described in following section.

\section{Polarity and motility of polymer-actin complex ge ${ }^{[15]}$}

An arrowhead-like pattern can be observed by TEM when HMM was decorated on native F-actin (Figure 5a), indicating that F-actin shows a well-defined polarity by self-organization. The pointed end of arrowhead and the opposite end of arrowhead are called the pointed end (P-end) and barbed end (B-end), respectively. To evaluate the polarity of actin complexes, we attempted to decorate the polymer-actin complexes with HMM by the same method used for F-actin. However, HMM was decorated only on the surface of the complex. Besides, the surface of the complexes was covered with myosin aggregates that are expected to be formed with extra polycations existing in the buffer solution. By adding polystyrene particles of $1.053 \mu \mathrm{m}$ in diameter, we successfully removed the extra polycation and obtained clear arrowhead images of polymer-actin complexes decorated with HMM. Figure 5a shows some examples of TEM images of HMM-decorated PDMAPAAQ-actin complexes and 6,4-ionene-actin complexes. Different from native F-actin that is a single strand, the polymer-actin complexes are bundles that consist of 3-20 filaments. Arrowhead structures within a filament of the bundle pointed in the same direction, although some defects are observed occasionally. However, arrowhead directions of filaments within a bundle are not completely the same. The complex polarity was estimated by:

$$
\text { polarity }=\frac{\left|n_{1}-n_{2}\right|}{n_{1}+n_{2}} \quad \text { (Eq.1) }
$$




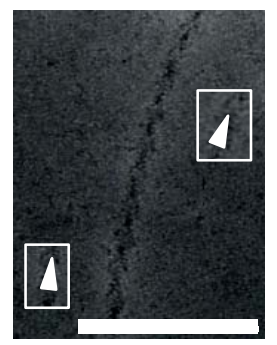

Native actin

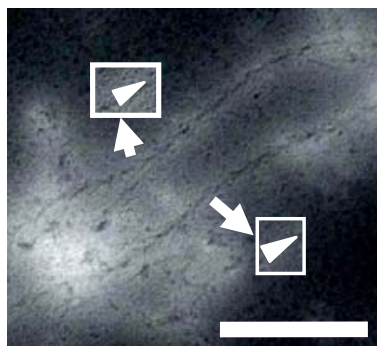

PDMAPAA-Q

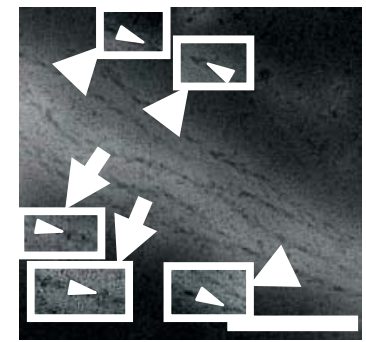

6, 4- ionene

(a)

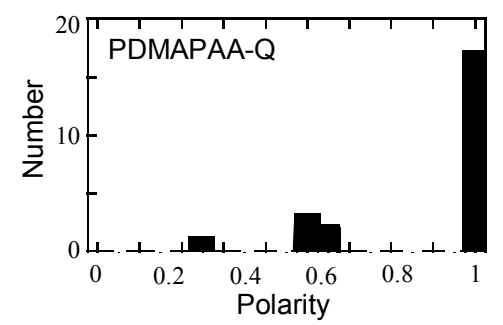

(b)

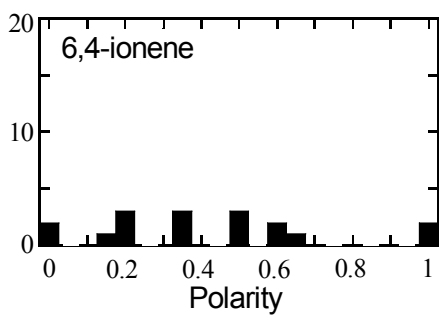

Figure 5 (a) Polarity of polymer-actin complexes decorated with HMM (Scale bars: 200nm). White arrows indicate the direction of arrowhead structures of decorated filaments. (b)

Histograms of complex polarity distributions. Cited from ref. 15 and asking for permission.

Table2 Polarities of polymer-actin complexes. Cited from ref. 15 and asking for permission.

\begin{tabular}{cc}
\hline Polymer & Polarity \\
\hline PDMAPAA-Q & $0.89(\mathrm{n}=23)^{*}$ \\
p-Lys & $0.76(\mathrm{n}=21)$ \\
3,3-ionene & $0.50(\mathrm{n}=22)$ \\
6,4 ionene & $0.42(\mathrm{n}=17)$ \\
6,10 ionene & $0.49(\mathrm{n}=22)$ \\
\hline
\end{tabular}

$*_{n}$ is the number of samples for the average.

Figure $5 \mathrm{~b}$ shows the distribution of the polarity of polymer-actin complexes. As shown in Figure 5b, PDMAPAA-Q complexes show a preferential polarity. In contrast to this, distribution of 6,4-ionene complexes are isotropic. The average polarity of the actin complex is shown in Table 2. As shown in Table 2, polarity depends on the chemical structure of polycations, and PDMAPAA-Q-actin complex shows the highest polarity as 0.89 (average over 23 samples), while 6,4actin shows the lowest value as 0.42 (average over 17 samples).

Thus, PDMAPAA-Q induces the formation of actin bundle having a 
unipolarity. These results show that actin and polycation form complexes with a preferential polarity.

To examine the correlation between the polarity and the velocity in polymer-actin complexes, the motility assays of p-Lys-actin complex, PDMAPAA-Q-actin complex gels and $\mathrm{x}, \mathrm{y}$-ionene $(\mathrm{x}=3$ or $6 ; \mathrm{y}=3,4$, or $10)$-actin complex gels in the presence of $4 \mathrm{mM}$ ATP were performed. We found that all these polymer-actin complex gels show a sliding motion. The average velocities of the PDMAPAA-Q-, 3,3-, 6,4-, and 6,10-ionene-actin complex gels, calculated from the mean displacement in $3.3 \mathrm{~s}$ over $17-39$ samples, were $1.13 \mu \mathrm{m} / \mathrm{s}, 1.3 \mu \mathrm{m} / \mathrm{s}, 0.69 \mu \mathrm{m} / \mathrm{s}, 0.48$ $\mu \mathrm{m} / \mathrm{s}$, and $0.79 \mu \mathrm{m} / \mathrm{s}$ respectively, which are comparable with those of native F-actin $(0.77 \mu \mathrm{m} / \mathrm{s})$. Among all these polymer-actin complexes, the PDMAPAA-Q-actin complex, which has the highest polarity, also shows the highest motility with a velocity of $1.3 \mu \mathrm{m} / \mathrm{s}$. Dendritic complexes, which are occasionally observed when F-actin is mixed with 6,4-ionene, did not exhibit a translational motion but instead migrate around their barycentric position. As shown in Figure 6, a linear relationship between polarity and velocity at $3.3 \mathrm{~s}$ was observed, that is. the velocity of the complex is proportional to the polarity. These results indicate that the polarity of the polymer-actin complex would dominate motility of polymer-actin complexes. A native F-actin moves toward one direction without moving back to the opposite direction. The polarity of F-actin motion determines the direction of its motility ${ }^{[19]}$. The reason that the polarity of polymer-actin complexes is correlated

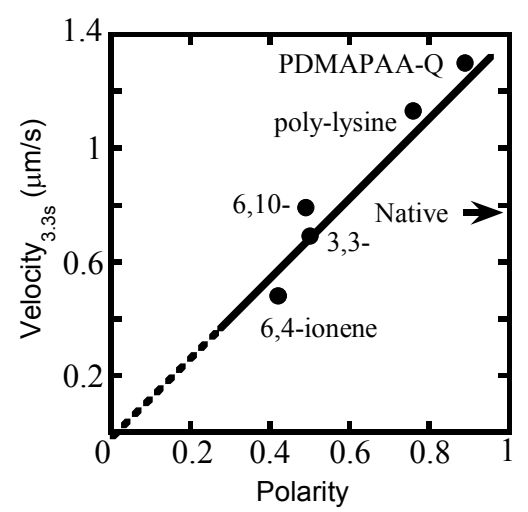

Figure 6 Relationship between the polarity and the sliding velocity as determined by a time interval of $3.3 \mathrm{~s}$ for polymer-actin complex gels. Velocity data are the average over 17 samples. The velocity of a native F-actin is shown by the arrow in the figure. Cited from ref. 15 and asking for permission. 
with its velocity can be explained as follows. For the polymer-actin complexes that are organized by F-actin, the whole polarity is determined by the polar direction of $\mathrm{F}$-actin. If $\mathrm{F}$-actins are assembled into polymer-actin complexes in an anti-parallel way, the offset effect of the whole polarity leads no sliding motion.

To characterize the sliding motion, the displacement $(d)$ of polymeractin complex gels and native F-actin was plotted on a logarithmic scale as a function of observation time $(t)$ (Figure 7). Data is an average over 17 samples. The displacement $(d)$ increases with the increase in the observation time $(t)$ and follows a power law as $d \propto t^{\beta}$. The exponent $\beta$ characterizes the motion. $\beta=0.5$ corresponds to random motion, while $\beta=1$ to constant linear translational motion. The $\beta$ values for p-Lys-, PDMAPAA-Q-, and 3,3-, 6,4-, 6,10-ionene-actin complexes, and native F-actin were $0.81,0.90,0.82,0.71,0.84$, and 0.69, respectively (Table 3 ). This shows that all polymer-actin complexes have more translational motion than that of native F-actin. Because polymer-actin complexes are thicker bundles than F-actins, they are less flexible than native F-actin due to the increase of the thickness. The

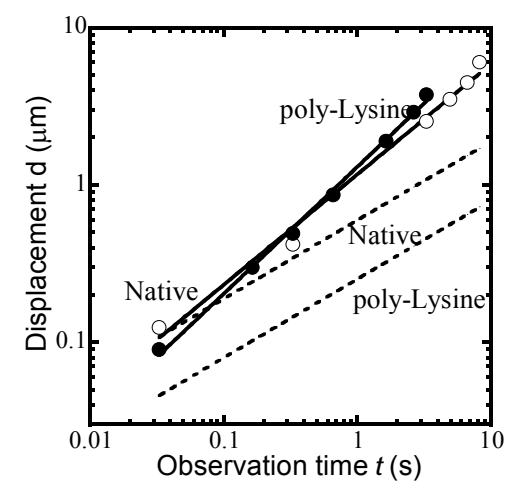

Figure 7 Logarithmic plot of displacement (d) of p-Lys-actin gel $(\bullet)$ and native F-actin $(\circ)$, as a function of observation time $(\mathrm{t})$. The solid lines are experimental observations, and the dotted lines are theoretical values supposing a Brownian motion. Cited from ref. 15 and asking for permission.

Table 3 Exponents $\beta$ of native actin and polymer-actin complexes. Cited from ref. 13 and asking for permission. Cited from ref. 15 and asking for permission.

\begin{tabular}{ccccccc}
\hline Polymer & native & $\begin{array}{l}\text { PDMA } \\
\text { PAA-Q }\end{array}$ & p-Lys & $3,3-$ & $6,4-$ & $6,10-$ \\
\hline$\beta$ & 0.69 & 0.90 & 0.81 & 0.82 & 0.71 & 0.84 \\
\hline
\end{tabular}


less random motion of polymer-actin complexes is attributed to the decrease of flexibility by bundling effect.

It has been known that F-actin motion on the glass surface coated with mysion shows that the sliding velocity is proportional to the probability, $f$, that one motor exerts the sliding force. $f$ is expressed as $f=\tau_{s} / \tau_{c}$, where, $\tau_{s}$ and $\tau_{c}$ are the time the sliding force is exerted on actin and the cyclic time of ATP hydrolysis, respectively ${ }^{[20,21]}$. The fact that a highly oriented actin bundles show a higher sliding velocity than that of a single F-actin indicates that if many motors act on the same actin bundle, fluctuation which is associated to the stochasticity of the motor is negligible.

It should be emphasized that the sliding motion observed in our experiment is different from Brownian motion. Sliding motion occurs two-dimensionally on the surface of the motor protein of myosin, whereas Brownian motion is three-dimensional thermal diffusion. It is tempting to make a comparison between the sliding velocity driven by the chemical energy of ATP and the diffusion velocity arising from thermal agitation. We estimate the diffusion coefficient, $D$, of the polymer-actin gel and native F- actin using the theory for stiff rods model $^{[22]}$ :

$$
D=\frac{k_{B} T}{2 \pi \eta_{s} L_{C}} \ln \frac{L_{C}}{b}
$$

where $b=12.1 \mathrm{~nm}$ and $25.3 \mathrm{~nm}$ are the average diameter of the native F-actin and polymer-actin gel, respectively. $L_{C}=2.13 \mu \mathrm{m}$ and $7.22 \mu \mathrm{m}$ are the average contour lengths of the native F-actin and polymer-actin gel, respectively, which are estimated from TEM and scanning electron micorscopy images. $k_{B}$ is the Boltzmann constant, $T$ is the temperature, and $\eta_{s}$ is the viscosity of the buffer. The diffusion coefficients of the native $F$-actin $\left(D_{a}\right)$ and polymer-actin gel $\left(D_{g}\right)$ estimated from eq. 2 are $18.8 \times 10^{-14}$ and $6.08 \times 10^{-14} \mathrm{~m}^{2} / \mathrm{s}$, respectively. As a result of its larger size of polymer-actin gel, the $D_{g}$ is about $1 / 3$ of the $D_{a}$; that is, native F-actin diffuses a $3^{0.5}$ times longer distance per unit time, as shown in Figure 7. For an observation time longer than 1s, for example, the motility of polymer-actin gel shows the velocity about 1 order of magnitude faster than that of the thermal diffusion.

Our results show that the cooperative motion of the polymer-actin complex is strongly dependent on the polarity of the complex. Thus, the further studies about making polymer-actin complex with same polarity are required for creating biomachine with high efficiency. 


\section{Acknowledgements}

This research is financially supported by SORST, JST and the Ministry of Education, Science, Sports, and Culture, Japan (Grand-in-Aid of Creative Scientific Research).

\section{References}

1. Huxley, H. E. (1969) The Mechanism of Muscular Contraction. Science. 164: 1356-1365.

2. Huxley, A. F. (1980) Reflections on Muscle. Liverpool University Press.

3. G. H. Pollack, (2001) Cells, Gels and the Engines of Life. Ebner and Sons Publishers, Seattle.

4. Kitamura, K., Tokunaga, M., Iwane, A. H., Yanagida, T. (1999) A single myosin head moves along an F-actin with regular steps of 5.3 nanometres. Nature. 397: 129-134.

5. Osada, Y., Okuzaki, H., Hori, H. (1992) A polymer gel with electrically driven motility. Nature. 355: 242-244.

6. Mitsumata, T., Ikeda, K., Gong, J. P., Osada, Y. (1998) Solvent-Driven Chemical Motor. Appl. Phys. Lett. 73: 2366-2368.

7. Osada,Y., Matsuda, (1995) A. Shape memory in hydrogels. Nature. 376: 219.

8. Osada, Y., Ross-Murphy, S. B. (1993) Intelligent gels. Sci. Am. 268: 82-87.

9. Jager,E. W. H., Smela, E., Inganas, O. (2000) Microfabricating Conjugated Polymer Actuators. Science. 290: 1540-1546.

10. Beebe, D. J., Moore, J. S., Bauer, J. M., Yu, Q., Liu, R. H., Devadoss, C., B-H. Jo. (2000) Functional hydrogel structures for autonomous flow control inside microfluidic channels. Nature. 404: 588-590.

11. Quake, S. R., Scherer, (2000) From Micro- to Nanofabrication with Soft Materials. Science. 290: 1536.

12. Kakugo, A., Sugimoto, S., Gong, J.P., Osada, Y. (2002) Gel Machines Constructed from Chemically Cross-linked Actins and Myosins. Adv. Mater. 14,(16):1124-1126.

13. Kakugo, A., Shikinaka, K., Matsumoto, K., Gong, J.P., Osada, Y. (2003) Growth of Large Polymer-Actin Complexes. Bioconjugate Chem. 14,(6):1185-1190.

14. Kakugo, A., Sugimoto, S., Shikinaka, K, Gong, J.P., Osada, Y. Characteristics of chemically cross-linked myosin gels. J. Biomater. Sci. Polymer Edn,. 16 (2) : 203-218

15. Kakugo, A., Shikinaka, K., Takekawa, N., Sugimoto, S, Osada, Y, Gong, J. P. Polarity and Motility of Large Polymer-Actin Complexes. Biomacromolecules. 6 (2): 845-849

16. Hayakawa, K., Santerre, J. P.; Kwak, J. C. (1983) Study of surfactant-polyelectrolyte interactions. Binding of dodecyl- and tetradecyltrimethyl- ammonium bromide by some carboxylic polyelectrolytes. Macromolecules. 16: 1642-1645.

17.Gong, J. P., Mizutani, T., Osada, Y. (1996) A comparative study on the cooperative binding of surfactants with solubilized polymers and networks. Polym. Adv. Techol. 7: 797-804.

18. Satake, I., Yang, J. T. (1976) Interaction of sodiumdecyl sulfate with poly (L-ornithine) and poly (L-lysine) in aqueous solution. Biopolymers. 15: 2263-2275.

19. Pollack, G. H., (2001) Cells, Gels and the Engines of Life, Ebner and Sons Publishers, Seattle.

20. Uyeda, T.Q.P., Korn, S.J., Spudich, J.A. (1990) Myosin Step Size Estimation From Slow Sliding Movement of Action Over Low Densities of Heavy Meromyosin. J. Mol. Biol. 214: 699-710.

21. Harada, Y., Sakurada, K., Aoki, T., Thomas, D.D., Yanagida, T. (1990) Mechanochemical Coupling in Actomyosin Energy Transduction Studied by in Vitro Movement Assay. J. Mol. Biol. 216: 49-68.

22. Doi, M., Edwards, S. F. (1980) The Theory of Polymer Dynamics 2nd edn. Pergamon, Oxford. 